
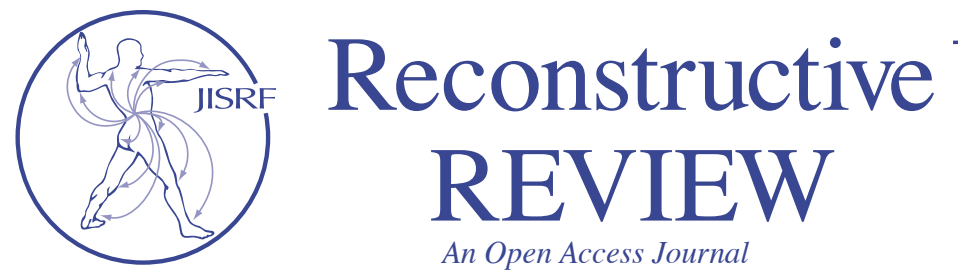

\title{
Year of the Mask \\ COVID-19 Challenges for Orthopaedic Surgery
}

\author{
Smith $E^{1}$
}

\section{November 2019 a novel coronavirus emerged in Wuhan, Hubei, China}

As a response to the viral pneumonias and severe illnesses that were emerging in patients, an ophthalmologist Dr Li Wenliang, working at Wuhan Central Hospital, voiced his concerns only to be severely admonished by the authorities. The accelerated spread of the Severe Acute Respiratory Syndrome (SARS) in Wuhan, and then globally, as a result of the novel coronavirus was acute and pronounced. China alerted the World Health Organisation to several pneumonia cases at the end of December 2019 and the first death was recorded in early January 2020 . The respiratory physician Dr Nanshan Zhong, announced human-to-human spread and a few days later on the 23 January 2020, Wuhan was placed under quarantine. The virus spread outside China and the WHO declared the outbreak a global health emergency on 30 January 2020. Tragically Dr Li Wenliang died on 7 February 2020 as a result of exposure to the virus, leaving a five-year-old son and a pregnant wife.

On 11 February 2020, WHO named the novel viral pneumonia as Coronavirus disease 2019 (COVID-19). The International Committee on Taxonomy of Viruses suggested the name 'SARS-CoV-2' as a result of their phylogenetic and taxonomic analysis of the virus.

Coronaviruses belong to the family of Coronaviridae, and comprise of large, single, plus-stranded RNA with a 29,903 nucleotide genome. There are 4 genera (designated $\alpha, \beta, \gamma, \delta)$ of coronavirus and $\beta$-CoV mainly infects the respiratory, gastrointestinal, and central nervous system of humans and mammals. 2019-nCOV is the 7th member of the family of coronaviruses. SARS-CoV and MERS-CoV also belong to $\beta-\mathrm{CoV}$ and the nucleotide sequence similarity between SARS-CoV and $2019-\mathrm{nCoV}$ is about $79 \%$.

SARS-CoV-2 possesses the typical coronavirus structure with a spike $(\mathrm{S})$ protein in the membrane envelope. This $\mathrm{S}$ protein can bind to the receptors of the host to facilitate viral

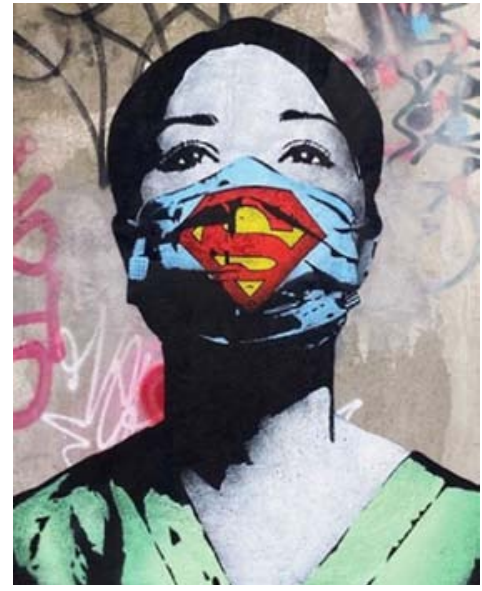

Street Art by FAKE; Amsterdam, Netherlands. Nurse with a mask with a Superman logo. entry into target cells and can also bind to the human angiotensin converting enzyme 2 (ACE2), but cannot bind to the human cells without ACE2. The high affinity between ACE2 and the $S$ protein also suggests that the population with higher expression of ACE2 might be more susceptible to SARS-CoV-2. [1]

It is highly likely that the virus originated in its natural host, the horseshoe bat (Rhinolophus affinis) and spilled out via some wild animals such as pangolins, and from a seafood and meat market into humans. The human to human transmission of the virus is via direct transmission (cough, sneeze, droplet dispersal and droplet inhalation) and contact transmission via oral, nasal and eye mucous

Keywords: Coronavirus; COVID-19; orthopaedic; surgery Level of Evidence: $V$ 
membranes. Although common clinical scenarios do not include eye symptoms, it has been shown that transmission of the virus is not limited to the respiratory tract, and includes eye exposure and direct contact with blood, oral fluids and other patient materials.

The spectrum of COVID-19 ranges from a mild selflimiting respiratory tract illness, to acute respiratory distress, severe progressive pneumonia, abnormal coagulation, inflammatory heart disease, neurological deficits, multi-organ failure, and death.

\section{The global spread of the virus produced a pandemic of respiratory illness involving 187 countries worldwide}

The timeline of COVID-19 is well known to the significant majority of people, as the world closed for business.

The WHO-China Joint Mission report on Coronavirus Disease 2019 (COVID-19) was published on the 29 February 2020. [2] By late February, early March there was an acceleration in the number of infected cases and the number of deaths due to the virus. The infection peak has now passed in Wuhan and there is a semblance of normality in the city. The first wave of COVID-19 seen in China has abated.

South Korea used extensive testing, isolation, and quarantines to keep its caseload low. Although the cap-

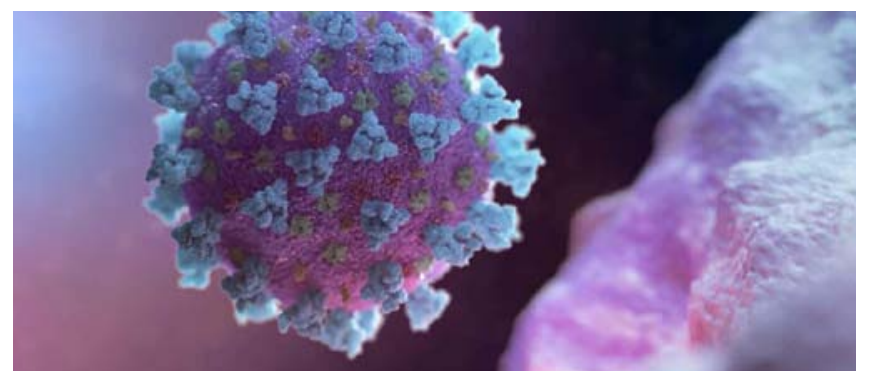

A model of a beta coronavirus, the virus linked to Covid-19. Photograph: NEXU Science Communication/Reuters from https:// www.theguardian.com/world/2020/apr/30/coronavirus-what-doscientists-know-about-covid-19-so-far. countries, particularly the USA, there were ponderous delays without any meaningful action.

National leadership failed to comprehend the devastation that lay ahead. Sadly, policy makers wished to control the narrative. It became clear that there was a schism between senior government officials and scientists, experts in the field of virology, epidemiology and public health and statisticians, due to inaccuracies and misrepresentation of the facts.

The inadequate testing for 2019-nCoV has been a huge issue, and this has impacted on the accuracy of the data to aid in the modelling of the pandemic. Furthermore, the lack of testing facilities has impacted on the ability to manage and contain the coronavirus pandemic.

The daily reports in the world media regarding the number of infections as well as the death rate, has fuelled the fear, hysteria and irrational behaviour in response to this worldwide disaster. A broad range of misinformation has spread across traditional media and social media in what WHO has called an infodemic (i.e. excessive amounts of misinformation, disinformation, and rumours that make it difficult to identify reliable sources of information). Removing false claims about COVID-19 and elevating authoritative information are welcome steps to help protect public health in this extraordinary time. $[\underline{3}]$

Risk of death for an individual person is unknown with COVID-19. The probability that someone dies from a disease does not just depend on ital, Seoul, is less than three hours from Wuhan by air, South Korea has had fewer cases and deaths than European countries such as France, Italy, Belgium or Spain.

The first South Korean case was confirmed on 20 January 2020, the same day as the first confirmed case in the United States of America (USA).

The first genome sequence of the virus from a human became available, from China, on 10 January 2020. South Korean officials responded and test kit mass production was initiated. Incisive action was seen in New Zealand which is also protected by its geographical position, while Sweden took an alternative light touch view on the 'lockdown' approach, compared with all the other countries. The 'lockdown' process occurred at different times in the various nations.

Strategic resources and medical supplies across the world were in short supply. In a number of first world the disease itself, but also on the patient's own ability to recover from it, on medical resources, and on the type and quality of the treatment they receive. The impact of obesity and age has been critical in the recovery of hospitalised patients. Immunocompromised patients, the elderly and those with comorbidities, such as cardiovascular disease, diabetes, obesity, chronic respiratory disease, hypertension and cancer, are at the greatest risk of dying. Countries with a more elderly population group will be the hardest-hit.

Black people and those from Bangladeshi, Pakistani, Indian, and mixed ethnicities have been shown to have a statistically significant increased risk of death from COVID-19 as compared with those of white ethnicity. The increased mortality is believed to be partly a result of socioeconomic disadvantage and other circumstances, but is yet to be fully understood. [4] 


\section{It is important to know about, and to understand, the difference between the Case Fatality Rate and Infection Fatality Rate}

Epidemiologists tend to use the Case Fatality Rate (CFR) or confirmed Case Fatality Rate (cCFR). CFR is the number of deaths from the disease divided by the number of confirmed/diagnosed cases of disease (preferably by nucleic acid testing). CFR is not constant and varies with location, country and time.

The Infection Fatality Rate (IFR) is the number of deaths from a disease divided by the actual/total number of cases of the disease/infection. The total number of COVID-19 is not known, because not everyone suspected of the disease is tested. With COVID-19 there are many undiagnosed cases worldwide.

When there are people who have the disease but are not positively diagnosed by having a specific test (as with mild and asymptomatic cases), the IFR is likely to be lower than the CFR.

Other recent pandemics such as the 2013-2016 outbreak of Ebola identified that the CFR was 40\%. [] ] The CFR for the MERS-CoV was 34\%, whereas the CFR for SARS-CoV was $10 \%$. []

The instantaneous reproduction number $R t$ of $\mathrm{COV}$ ID-19 is analysed so that control measures can be regularly assessed and monitored during the lockdown phase. The $R t$ is defined as the average number of secondary cases generated by one primary case with symptom onset on day $t$. If $R t>1$ the epidemic is expanding at time $t$, whereas $R t$ $<1$ indicates that the epidemic size is shrinking at time $t$. At the stage where $R t<1$, control measures may be safely relaxed.

'Lockdown' measures have flattened the epidemic curve but flattening the curve does not mean that infected cases disappear, merely that the number of COVID-19 cases are spread over a longer time period allowing healthcare systems to cope. It is unknown whether deferring infected cases will prove advantageous. However, close monitoring of $R t$ and cCFR is necessary to inform strategies against a potential second wave, to achieve an optimal balance between health and economic protection.

As the COVID-19 response gathers momentum across sub-Saharan Africa, additional research will be needed to fully understand the susceptibility, transmission dynamics, pathogenesis and clinical outcomes of COVID-19 among people living with HIV compared to the general population.

On 23 March 2020, the 'lockdown' phase (Level 4 National Incident) started in the United Kingdom (UK). Social distancing and symptom positive self-quarantine was effected. Only essential services remained operative, online shopping and delivery services were permitted. Once daily outdoor exercising within one's locality, travel to/ from work, if unable to work from home, and travel to buy basic necessities like food or medicines were allowed. Social visits, gatherings and all sport was cancelled, Schools (except for vulnerable pupils and children of key workers). Universities were closed. Final year medical school exams were suspended. Social distancing was enforced everywhere.

Measures altering population behaviour and social distancing substantially reduced virus transmission. However, it was necessary to adopt a functional working framework in key labour markets during 'lockdown' to enable working from home, physical interface for essential travel, and to accommodate those working away from home.

The National Health Service (NHS), at risk of being overwhelmed, geared up and prepared for the increased burden of admissions and severely ill patients, many of whom would require intensive care support as a result of COVID-19.

The regular NHS service was suspended, resources and staff were redeployed to cope with the influx of daily patients into hospitals all over the country. Visitors to hospital were restricted and detailed coronavirus supporting information was provided to staff, patients and the few permitted visitors.

Elective orthopaedic surgery was halted and many consultant orthopaedic surgeons' roles were sublimated to provide support on wards and to undertake work in minor fracture clinics. Orthopaedic consultants acted as the third wave of support behind the orthopaedic residents and junior doctors. Personal protective equipment (PPE) training was provided, resuscitation skills refreshed, and up skilling to provide care on intensive care units (e.g. proning patients, use of ventilators) was widespread. Retired doctors were requested to return for duty. Heads of departments and senior consultants in managerial roles as 'directors', were involved in strategic organisation of the major hospitals and organisation of acute response programs to deal with the predicted tsunami of patients at the peak of coronavirus pandemic transmission.

Frontline healthcare workers are at high risk of infection, in particular Anesthesiologists, Ear Nose and Throat Surgeons, and Intensive Care Specialists and nurses. Tragically, there were a number of deaths of healthcare workers in many world countries.

Alarms were raised concerning PPE shortages in the $\mathrm{UK}$ and this is assessed and addressed on a frequent basis. Up to date guidance during the pandemic is regularly circulated by the Royal College of Surgeons of England. [ $[\underline{8}, \underline{8}]$ 
Trauma and emergency admissions have reduced by some $40 \%$ in the UK. However, surgical activity and front line orthopaedic care continued with acute trauma, orthopaedic cancer and desperate cases during 'lockdown'. Conservative treatment of fractures was adopted wherever feasible and imaging reduced, except where vital.

Use of masks by patients is not mandatory, but hospital facilities have adjusted for social distancing, hand hygiene facilities and protective screens are used where feasible.

Nasopharyngeal swab tests for SARS-CoV-2 and low dose CT scans which accurately evaluate the lung fields, are performed to assess the presence of COVID-19 infection prior to surgery.

\section{Algorithms for treatment for asymptomatic versus symptomatic patients have been produced}

There are unresolved issues regarding risk disclosure and consent for orthopaedic operations during the pandemic. It is possible that the USA and UK, who experience the highest and similar negligence claims, may be most affected. Informed consent for orthopaedic operations is crucial in the surgeon-patient relationship and it would be helpful for a unified approach to this potential problem during, and beyond, the pandemic.

Protocols have been individualised for the different institutions regarding the flow of traffic in the operating room (OR) complex, OR staffing levels, equipment volume, patient transport to and from the OR and post-operative care facilities.

Patients are anaesthetised, intubated and extubated in the OR.

All OR staff involved in patient care wear full PPE. An N95 respirator mask (or equivalent), full-face shield or safety goggles, fluid resistant gown, double gloves, and a hat or surgical balaclava is standard practice.

Power tools used in drilling, reaming, cutting and impaction of bone and electrocautery in the OR, facilitate the spread of droplets from mechanical disruption, and this supports the spread of SARS-CoV-2 virus. While difficult in modern orthopaedic surgery, minimisation of aerosol generating activity is important.

The high airborne virus load places the operating orthopaedic surgeon, and all surgical staff in the OR at high risk. This risk is far greater than in the community, where the viral load would be lower.

Enhanced pre-operative and post-operative preparations and cleaning, delays to allow for clean air exchange in the OR post extubation and between surgeries, has ex- tended the average duration for all procedures undertaken. Necessary radiographs are performed in the OR wherever possible. Wound closure is performed in the standard fashion and the wound sealed with occlusive dressing.

The doffing of PPE gear is critical. It is performed with care and attention to detail, in designated areas and is followed by thorough hand washing, prior to leaving the theatre complex. The OR is cleaned with chlorine based or similar disinfectant to eliminate the virus on surfaces.

During my specialist orthopaedic training in South Africa, orthopaedic patients requiring surgery were assumed to be HIV positive. A standard approach to surgical procedures was always adopted, with the maximum vigilance and protection available. The same strategy is mandatory in orthopaedic surgery during the pandemic, and beyond.

Post-operative care regimes have already adapted and altered to allow for early discharge with post-operative follow-up and rehabilitation by remote consultation if possible. Venous thromboprophylaxis is essential except for those with a known aberrant response to such treatment. Specific arrangements are made with regard to operative complications.

Prior to the pandemic, the Internet had fundamentally changed the way individuals were managing healthcare. Numerous Apps for healthcare professionals already exist. Telemedicine, social media and online technology utilised in the retrieval of meaningful information now become more essential than ever. Virtual medicine and working in 'bubbles' as in New Zealand, has been harnessed in the care of patients.

Population demographics, the needs of orthopaedic patients and medical resources differ throughout the world. The review for resuming elective orthopaedic surgery during the COVID-19 pandemic provides extensive and detailed guidance, but at the same time a utopian consensus on this subject. [9] Orthopaedic surgeons have already adapted their approach and response to the pandemic in daily trauma operating based on their national and local predicament.

\section{In all probability, the trauma burden in the UK will increase as relaxation of 'lockdown' takes place}

Furthermore, as the UK overcomes the peak of the first wave of the pandemic, the NHS will prepare for the resumption of elective cases in hospitals across the country, in light of the potential second wave of COVID-19, predicted by many international virologists. Increases in demand and changes to supply will not only affect patients 
with COVID-19 but will have massive knock-on effects on care provided to the wider population. This challenge will reverberate throughout the majority of the 187 countries affected by the pandemic. It is also likely that the changes will impact most on the poorest socio-economic groups. Inevitably NHS waiting times for elective care will rise.

From an ethical perspective, there is little doubt that patients thought to be infected, or at high risk from COVID-19, should have elective surgery deferred and also receive Reverse Transcriptase Polymerase Chain Reaction (RT-PCR) testing. Parvis et al. identify no evidence to support routine serum antibody testing for all patients, but following the development of a vaccine, antibody testing will be crucial to differentiate at risk populations.

\section{RT-PCR testing of saliva and sputum should ultimately be part of mandatory screening for patients undergoing elective surgery}

High Efficiency Particulate Air filtration (HEPA) systems should be utilised in the OR as well as in more crowded areas in hospitals. [9] Periodic surveillance in hospitals should become part of an institutions' protocol to halt the unknowing spread of this, or any future devastating virus.

Ultimately a proportion of general population will acquire immunity against SARS-CoV-2, which mutates at a lower frequency as compared with seasonal influenza virus. There is intense worldwide research for an effective treatment to counter COVID-19 and many institutions and pharmaceutical companies are performing research to develop a vaccine. It is hoped that a vaccine may be available in early 2021. It is equally possible, however, that it will be many years before global collaboration brings fruition and delivers the Holy Grail.

The burden of the pandemic is unquantifiable, both to the world economy, where choices are already being made regarding survival versus sickness, as well as the impact on world healthcare. The 'lockdown' has resulted in mental health deterioration, reduced exercise, domestic and child abuse, increased alcohol use, distress, fear and in some cases death. The future healthcare challenges will be immense.

As in all times of threat, there has been churlish, inappropriate and despicable behaviour, but this is contrasted by great human kindness, endeavour and support, especially for the vulnerable and elderly, as well as the virtual sharing seen on a daily basis throughout our interactive world.

As a result of the COVID-19 pandemic there has been a seismic paradigm shift in life as we know it, and this will resonate for decades to come. It has impinged upon all our lives and it is a challenge for all orthopaedic surgeons worldwide.

A happy irony however is that it has brought about the healing of the planet.

The Great Wall of China is no longer a fortress, Mecca is empty, Disneyland has no more magic and Paris is no longer romantic!

The safety of the patient remains paramount, but the safety of all healthcare workers is also vital. There is colossal appreciation for all front line and key workers in every sphere of life in 2020 - the year of the mask.

\section{References}

1. Peng X, Xu X, Li Y, Cheng L, Zhou X, Ren B. Transmission routes of 2019-nCoV and controls in dental practice. IJOS 2020; 12: 1-6. https://doi.org/10.1038/s41368-020-0075-9

Report of the WHO-China Joint Mission on Coronavirus Disease 2019 (COVID-19). 16-24 February 2020. https://www.who.int/docs/default-source/coronaviruse/who-china-joint-mis$\underline{\text { sion-on-covid-19-final-report.pdf }}$

3. Limaye RJ, Sauer M, Ali J, Bernstein J, Wahl B, Barnhill A, Labrique A. Building trust while influencing online COVID-19 content in the social media world. The Lancet April 21, 2020. https://doi.org/10.1016/S2589-7500(20)30084-4

4. Office for National Statistics Coronavirus (COVID-19) Coronavirus (COVID-19) related deaths by ethnic group, England and Wales: 2 March 2020 to 10 April 2020. Last updated: 7 May 2020 https://www.ons.gov.uk/peoplepopulationandcommunity/birthsdeathsandmarriages/deaths/articles/coronavirusrelateddeathsbyethnicgroupenglandandwales/2march2020to 10 $\underline{\text { april2020 }}$

5. Shultz JM, Espinel Z, Espinola M, Rechkemmer A. Distinguishing epidemiological features of the 2013-2016 West Africa Ebola virus disease outbreak. Disaster Health 2016; 2016, 3: 78-88. http://dx.doi.org/10.1080/21665044.2016.1228326

6. Munster VJ, Koopmans M, van Doremalen N, van Riel D, De Wit E. A Novel Coronavirus Emerging in China - Key Questions for Impact Assessment. N Engl J Med 2020; 382: 692694. DOI: 10.1056/NEJMp2000929

7. Guidance for surgeons working during the COVID-19 pandemic. The Royal College of Surgeons of England. Published 20 March 2020. https://www.rcseng.ac.uk/coronavirus/jointguidance-for-surgeons-v1/

8. Updated Intercollegiate General Surgery Guidance on COVID-19. The Royal College of Surgeons of England. Published 26 March 2020. https://www.rcseng.ac.uk/coronavirus/jointguidance-for-surgeons-v2

9. Parvizi J, Gehrke T, Krueger CA, Chisari E, Citak M, Van Onsem S, Walter WL and other members of the International Consensus Group (ICM) and the Research Committee of the American Association of Hip and Knee Surgeons (AAHKS). Current Concepts Review Resuming Elective Orthopaedic Surgery During the COVID-19 Pandemic. Guidelines Developed by the International Consensus Group. J Bone and Joint Surg 2020; pages 1-43. (In Press-read on line). http://dx.doi.org/10.2106/JBJS.20.00844

SUBMISSION HISTORY

Submitted: May 10, 2020

Revised: May 20, 2020

Accepted: May 26, 2020

Published: July 14, 2020

AUTHOR AFFILIATIONS

1 Evert Smith, BSc MBBCh FRCS. Consultant Orthopaedic Surgeon, Spire Bristol Hospital \& The Glen, Redland Hill, Redland, Bristol BS6 6UT United Kingdom International Editor for the Joint Implant Surgery \& Research Foundation (JISRF) journal - Reconstructive Review

(Direct inquires to Evert Smith, evert@evertsmith.com)

AUTHOR DISCLOSURES

The Author declares that there is no conflict of interest.

COPYRIGHT \& OPEN ACCESS

(C) 2020 Smith. All rights reserved.

Authors retain copyright and grant the journal right of first publication with the work Reconstructive Review is an open access publication and follows the Creative Commons Attribution-NonCommercial CC BY-NC. This license allows anyone to download works, build upon the material and share them with others for non-commercial purposes as long as they credit the senior author, Reconstructive Review, and the Joint Implant Surgery \& Research Foundation (JISRF). An example credit would be: "Courtesy of (senior author's name), Reconstructive Review, JISRF, Chagrin Falls, Ohio". 